\title{
A framework for IoT-enabled environment aware traffic management
}

\author{
Mohamed Fazil Mohamed Firdhous ${ }^{1}$, B. H. Sudantha ${ }^{2}$, Naseer Ali Hussien ${ }^{3}$ \\ ${ }^{1,2}$ Department of Information Technology, University of Moratuwa, Sri Lanka \\ ${ }^{1,2}$ Center of Excellence in Intelligent Transpost Systems, University of Moratuwa, Sri Lanka \\ ${ }^{3}$ College of Education for Pure Sciences, Wasit University, Iraq
}

\begin{tabular}{l} 
Article Info \\
\hline Article history: \\
Received Feb 1, 202 \\
Revised Jul 7, 202 \\
Accepted Jul 25, 202 \\
\hline Keywords: \\
Congestion \\
Google maps \\
Health hazards \\
Internet of things \\
Pollution
\end{tabular}

Article Info

Article history:

Received Feb 1, 2020

Revised Jul 7, 2020

Keywords:

Congestion 
Presently more than half the world population lives in urban areas [4]. The proportion of urban population increases rapidly as most part of the world experiences rapid urbanization due to the advances in technology and movement of people towards cities searching for better life. By 2050, nearly 10 billion people, which would be around $66 \%$ of the world population at that time will be living in urban centers of the world [5]. The main reason for increased vehicles on the road and resulting traffic congestion is the unbalance transportation of supply and demand. Increasing road supply, that is building new roads to cater to the increased number of vehicles would be one kind of solutions to alleviate supply and demand contradictory. However, the experience from many countries indicates that the dependence on constructing more roads would inevitably result in the vicious cycle of further increasing the traffic congestion on roads. [6]. One of the effective ways of managing traffic especially during peak hours is the effective traffic management using traffic control systems [7]. Hence the experts in the field are required to come up with other methods of managing traffic in cities.

The above discussion underscores the requirement for alternative methods for managing and controlling traffic with special reference to reducing environment pollution in cities. In this paper, the authors present a conceptual framework for using internet of things (IoT) for managing and controlling traffic in a city with special emphasis on mitigating the impact of traffic on the environment. This paper is organized into four different sections as follows: Section 1 gives an overall introduction to the paper along with a brief analysis of traffic congestion prevailing in cities and the problems caused by them. Section 2 describes the methodology adopted in designing and development of the framework for the IoT enabled environment aware traffic management along with the background of Internet of Things in detail with special emphasis on the role of IoT in environment monitoring and traffic management. Section 3 presents the proposed framework in detail paying special attention to every finer point. Finally, Section 4 concludes the paper with a discussion on the importance of the framework presented along with suggestions for extending this framework to cover the entire spectrum of traffic management.

\section{RESEARCH METHOD AND RELATED WORK}

This section presents the research method used in this work in brief along with a detailed discussion on the relevant background work.

\subsection{Research method}

In order to carry out the proposed work in a scientific manner, an agile research and development methodology was devised and used. Figure 1 shows the main steps involved in the devised methodology. The research process started with the definition of the study area along with an extensive literature review on all the relevant areas with special emphasis on understanding all the required background theory and applications. Then it progressed gradually in an organized manner as shown in Figure 1. During the final stages of the study, the identified sub units were designed and implemented with special emphasis on modular design and testing. Finally the individual modules were integrated as single working units ready for testing in a simulated environment.

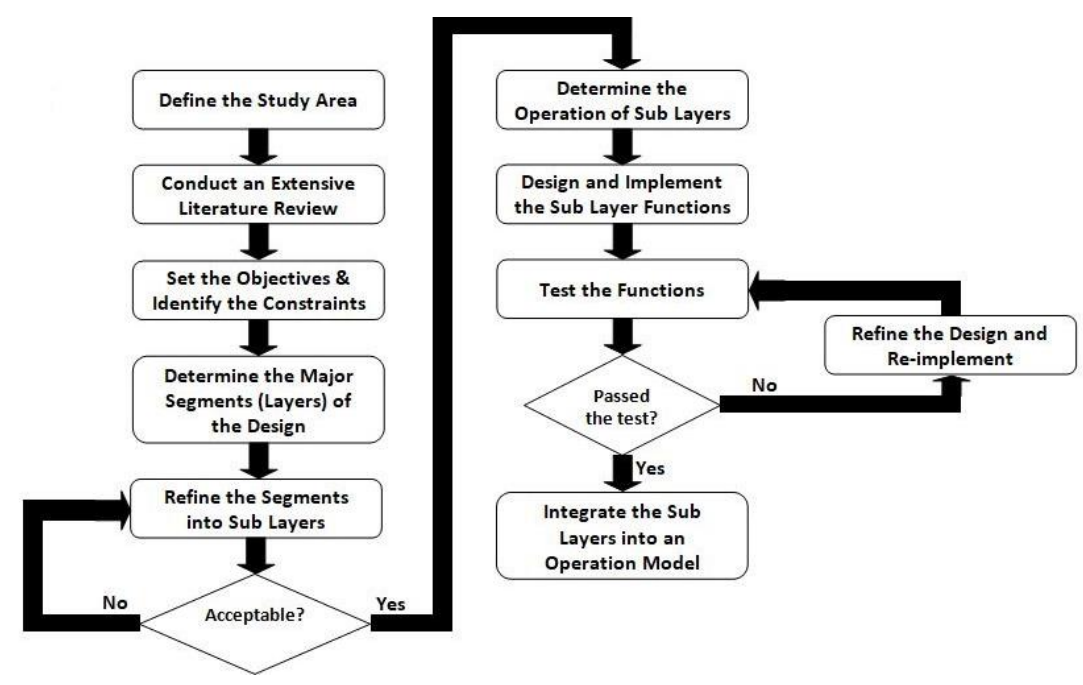

Figure 1. Research methodology 


\subsection{Related work}

This section briefly surveys the related work starting with a brief introduction to IoT and then an in depth look at its application in the areas of environment monitoring and traffic management. In order to present the works in an organized manner, this section is divided into three subsections. Subsection 2.2.1 presents IoT in a broader perspective with special emphasis on its features and capabilities following Subsections 2.2.2 and 2.2.3 discussing its application in environment monitoring and traffic management.

\subsubsection{Internet of things}

IoT enables the ordinary devices to become intelligent or smart and communicate with each other with objective of actively monitoring and managing the environment they are in [8]. The things in IoT can be either physical objects or virtual ones or both that can be connected together and communicate over the Internet for the purpose of sharing information and making intelligent decisions. The concept of IoT became feasible due to advancements made in many fields including electronics, sensor technologies, software systems and communication technologies. Other related fields where research has been carried out in parallel with the same objective of creating a smarter world include cloud computing (CC), fog computing (FG), mobile computing (MC), pervasive computing (PC), wireless sensor networks (WSN), and cyber physical systems (CPS) [9-12].

With IoT, the expanse of the Internet has been extended beyond computers as end nodes to real world objects including household appliances, vehicles and their components, environment monitors etc., [13]. IoT enables the ordinary real-world objects not only become intelligence but also communicate with other real world as well as virtual objects. The ordinary devices thus enhanced through the embedding of limited computing power, memory and communication capability in them are commonly known as smart objects irrespective of whether they are real or virtual. These smart objects are the foundation of IoT. These objects not only have the ability to sense and understand the environment they are placed in but also control the environment. The penetration of IoT into day to day life is evident that ordinary household equipment including sewing machines, exercise bikes, electric toothbrushes, washing machines, electricity meters and photocopiers are being computerized and equipped with network interfaces.

On the other hand, the Internet provides seamless connection enabling these devices to communicate with each other and control them from remote locations [14]. The ability to collect real time information enables the monitoring and control of the environment in detail that was previously unattainable at very low costs as these devices can be produced on mass scale. Along with the ability collect data in real time, the advances in data processing techniques such as big data analytics and deep learning, it is not only possible to understand underlying processes that are responsible for these changes, but also better control and manage these processes for the advantage of the controller. This ability to sense, understand and react to the events in the physical world in an automatic, rapid and informed manner opens up new avenues for dealing with complex or critical situations as well as enables a wide variety of business processes to be optimized. From the above discussion, it can be seen that IoT is multi-faceted due to its versatility and wide range of applications that can be built on them. The different facets of IoT are shown in Figure 2 graphically as individual elements.

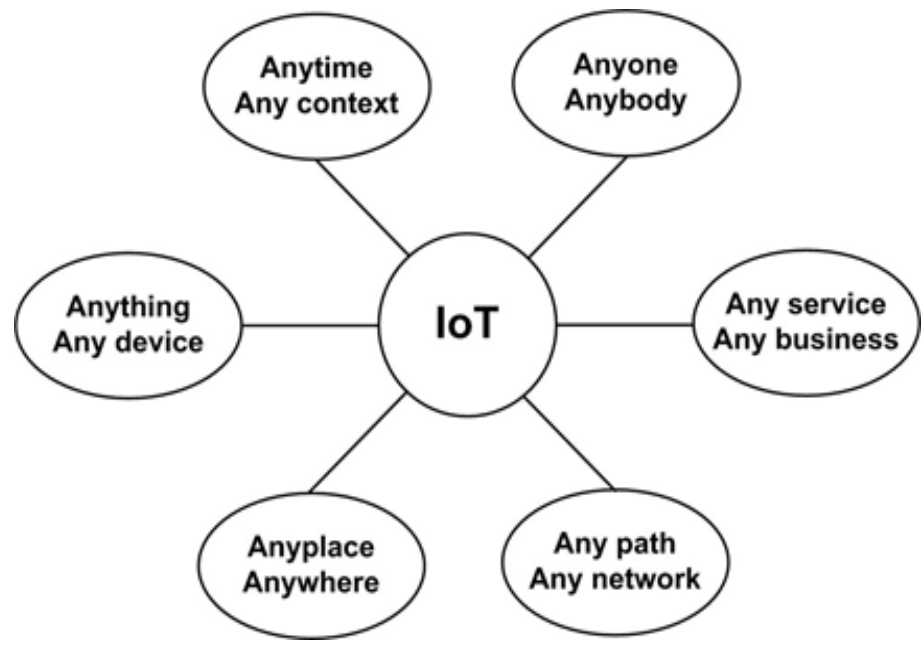

Figure 2. Elements of internet of things [15] 
IoT based applications have been implemented in many areas including environment monitoring and management, home automation, security systems, energy management in public and private spaces, elderly care systems and microclimate monitoring systems [16]. The IoT-powered environment aware traffic management proposed in this research would employ both semiconductor sensors and electromechanical transducers along with other supporting elements for effectively monitoring the relevant aspects of a chosen environment. The IoT device would be programmed to collect and transfer samples at regular intervals and transfer them to the central processing station through the Internet.

\subsubsection{Internet of things in environment monitoring}

There are many research work published in the literature on IoT in environment monitoring. But, many of them concentrate on monitoring the general environment where they mainly concentrate on drivers of climate change, green houses, industrial waste monitoring, short term temperature and humidity monitoring or indoor environment monitoring [17-21]. In this section, the authors take a brief look at the work carried out on traffic related environment monitoring reported in the literature with respect their strengths and weaknesses.

Kavitha et al. have in [22] presented a pollution monitoring system using IoT on Raspberry-PI. In the proposed system, the IoT based monitoring units collect the pollution data at various points and display those values on the web. The proposed system has no more application that displaying the raw data and hence has limited applications in practical situations. The IoT based carbon monoxide (CO) monitoring system for smart cities proposed by Paruchuri and Rajesh in [23] suffers from several shortcomings. This is only a survey of limited CO monitoring techniques and it does not provide a critical analysis of the techniques surveyed based on their capabilities and limitations. Hence, it is difficult to derive any practical benefits from the results presented in this work.

Firdhous and Sudantha have in [24] presented a microclimate monitoring system combining the strengths of both IoT and cloud computing. This work presents a novel technique for combining two advanced networking paradigms for exploiting their strengths as IoT has limited resources and capabilities while cloud computing systems theoretically unlimited resources. One the other hand, IoT devices can be installed in any place due to their miniature sizes and limited resource requirements including power and cloud systems demand high power and need large spaces to house high end computer systems. Hence, this work has high potential for extending into many application areas including monitoring and actuation.

Suparman and Jong have proposed an automatic smoke detection system using IoT in [25]. The main objective of this system is to inform the house owners in times of fire irrespective of their current location. Though at present levels, it cannot be used directly to manage air pollution caused by increased traffic on the road, it can be potentially enhanced to monitor and control environment traffic real time. The system proposed by Hawari et al. in [26] is capable of monitoring air quality based on Malaysia air pollution index (API) that employs an IoT network to build the monitoring and evaluation infrastructure. This low-cost system is capable of monitoring regular pollutants including Particulate Matter of PM2.5, PM10 and CO gas as well as the temperatures and humidity of the surroundings in real time. The system is also capable of categorizing the environment Good, Moderate, Unhealthy, Very Unhealthy and Hazardous based on API standards. Although, the system proposed by Hawari et al. in [26] has many advantages, it is only a monitoring system and is unable to take any effective action to improve the quality of the environment or reduce traffic congestion.

\subsubsection{Internet of things in traffic management}

Huang et al., have developed and tested a prototype IoT based wireless sensor system for traffic volume and vehicle classification using wireless accelerometers [27]. The proposed system is installed on roadway shoulder without interfering with the ongoing traffic to monitor pavement acceleration that will be used compute the number of vehicle axles, axle spacing and speed. The wireless sensors in the system can measure $\mathrm{X}, \mathrm{Y}$ and $\mathrm{Z}$ accelerations in the longitudinal direction along with tire movement in the transverse direction perpendicular to the movement and vertical directions respectively. The proposed system can only be used for identifying vehicle types based on the number of axles and axle spacing and compute the total number of vehicles under different groups. This system has only very limited application in managing the impact of the vehicular traffic on the environment.

Matharia and Dave have presented a smart traffic management system using IoT [28]. The proposed system uses vehicle mounted passive radio frequency identification (RFID) tag for identifying each vehicle uniquely and RFID readers mounted at the roadside at regular spacing. When a vehicle passes a roadside RFID reader the RFID tag mounted in the vehicle gets activated and transmits information including its ID to the reader, which in turn transmits it to the central processing center along with a time stamp. This information is used to compute the vehicle's speed and direction. When the system detects over-speeding vehicles it alerts 
both the driver of the vehicle and the highway patrol. The proposed system is very simple and can monitor vehicle movement with respect to their speeds only. Hence, it does not have any application in managing traffic with respect to the maintaining a livable environment near highways or other roadways.

Bhagchandani and Augustine have proposed an IoT based heart monitoring and alerting system with cloud computing and managing the traffic for an ambulance for India [29]. This system monitors the condition of the patient using an IoT enabled body area network and then uses that information to control the traffic lights on the way to hospital overriding the controls of the traffic signals. This is an adhoc control of signals rather than an objective management and control of traffic so that the negative impact of traffic at sensitive geographical locations is minimized. Hence, this method has limited or no generic applicability in managing and controlling traffic in given geographical locations.

Hashim et al., have in [30] proposed an automatic traffic light controller for emergency vehicle using peripheral interface controller that enables the emergency vehicle driver to control the traffic signals at intersections. The proposed mechanism employs a peripheral interface controller (PIC) to program a prioritybased traffic light controller for emergency vehicles and uses radio frequency (RF) to communicate with the traffic lights at the junction. The signal will return to normal operation once, the emergency vehicle completes crossing of the intersection. This technique is an adhoc traffic control technique that provides a selected set of vehicles the power to override signals at intersections and hence, this proposed system is not based on any objective criterion to manage traffic so that environment pollution or congestion is minimized. This s a very primitive approach and will not reduce the cumulative waiting time of all the other vehicles going through the given geographical location. Hence, this approach has very limited application in managing pollution/traffic at critical locations.

Malik et al., have in [31] described a methodology for efficiently managing traffic in highly populated and congested areas using IoT. The proposed framework employs decisive algorithm and round-robin algorithm to find the optimum path through traffic. IR sensors are used for determining total traffic in a region while RFID attached to every vehicle will enable system to detect high priority vehicles and route them fast. The main downside of the proposal is the requirement of every vehicle to have an RFID tag attached to them. The authors also claim that the RFID will help to detect robbed vehicles. When a vehicle is robbed, the first thing the robbers will do is disabling or removing such equipment from the vehicle. Hence, the proposed methodology has very limited application in real world scenarios.

Dzulkurnain et al., have in proposed an IoT based parking management system that help a driver to locate a free parking lot with relative ease [32]. Though the proposed method may theoretically help reduce the traffic in a given area, it practically has the shortcoming of increasing traffic in other areas. During peak times, every driver who could not find a suitable parking space would be looking for a parking spot in a given area that is already congested, and hence, every driver who has spotted a free slot may try to go towards that lot will create more traffic in that particular area. Hence, this method has very limited practical application in managing traffic in a real world scenario.

From the above discussion it can be seen that traffic management and pollution monitoring are handled separately by the researchers so far. In order to arrive at a practical solution managing the pollution created by vehicles is long overdue. This concept paper will be a starting point towards that integrated traffic and pollution management together on the same platform.

\section{PROPOSED IoT-ENABLED TRAFFIC MANAGEMENT FRAMEWORK}

This section presents the proposed ICT framework for the environment-aware traffic management system. The proposed framework is developed as a layered architecture as it has many advantages during implementation. The advantages of layered architecture include modularity, simplicity, maintainability, flexibility, scalability, portability, robustness and implementation stability with respect to adhoc implementations [33]. System developed based on the proposed framework will contain two main components. These two main components are: a set of sensing nodes installed at various strategic positions within a given geographical area and the other one if the central processing and knowledge generation unit (central control). The sensing nodes will collect the pollution data at the place, where they are installed in and the central control installed in a high end computer will analyze the data received from multiple sensing nodes and generate the dynamic pollution map of that area. Then, based on the current pollution levels at the predefined specific geographical areas such near schools, hospital or identified residential neighborhoods and the projected trends will determine if the pollution levels are within accepted limits or may go beyond these limits. When the pollution levels reach a predetermined threshold levels at the identified areas, the central control will start sending instructions to the vehicles advising them to take alternate routes avoiding the vulnerable locations. Figure 3 shows the overall architecture of the proposed system. 


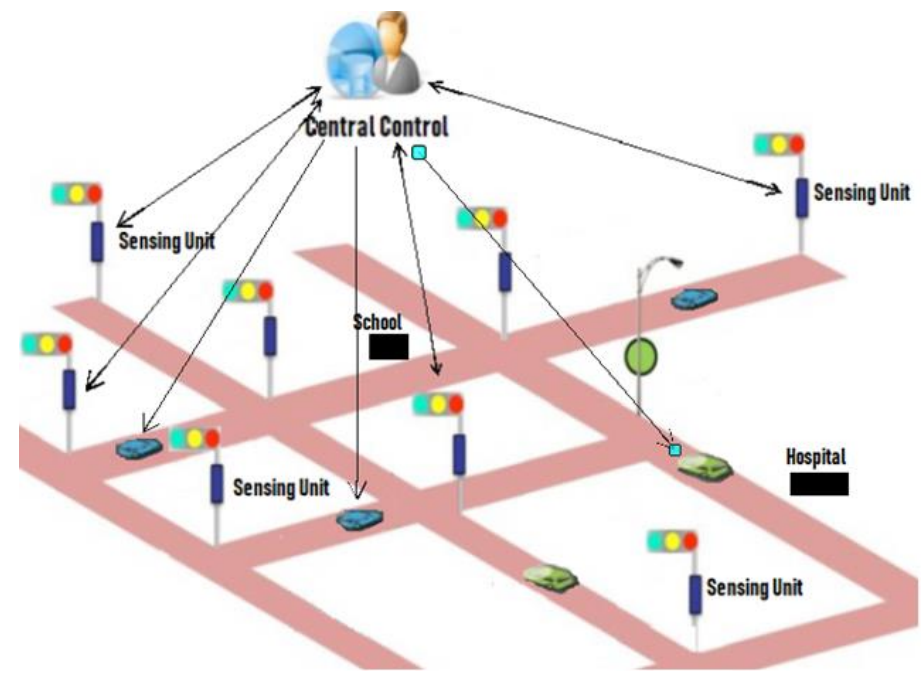

Figure 3. Architecture of the proposed system

The operation of the entire system has been designed as a layered framework for easy development, management and future enhancements. The different functions of the operation have been clearly defined and are to work within an identified functional layer. The layered operational model insulates the operation of each function from that of the other functions. Hence, each function can be modified independently without affecting the operation of other functions provided the data exchange interface between them is maintained unchanged. This will greatly facilitate the future enhancement of the overall system enabling to take the advantage of future developments without modifying the entire system. Figure 4 shows the proposed layered framework identifying the general functions to be within each layer broadly. The proposed framework consists of four main layers and each layer will be further subdivided into sub-layers and explained in the subsequent subsections.

\begin{tabular}{|c|}
\hline Knowledge Dissemination Layer \\
\hline Data Analysis and Knowledge Generation Layer \\
\hline Communication Layer \\
Data Acquisition Layer \\
\hline
\end{tabular}

Figure 4. ICT layered framework

\subsection{Data acquisition layer}

Successful development and implementation of effective traffic management systems demands high quality data collection in real-time [34]. The collected data should not only meet the time demands but also quality demands as small changes in certain parameters may produce big impacts on the environment. Thus, the data collection process must be capable of acquiring different types of data at different levels on accuracy and granularity. Mostly the environment parameters collected including traffic data tend to be analog in nature. Hence the data collection system must be capable of digitizing the data with the correct quantization and coding levels. Then the digitized data must be encoded in a suitable format for local storage considering the environment conditions.

Figure 5 presents the proposed model of the data acquisition layer. At the lowest level of the data acquisition layer will be made up of a distributed sensor network that will sense the different pollution parameters. The information collected by these sensors will be passed to a sampling and quantizing sub-layer which will in turn be encoded using an efficient encoding algorithm suitable for storage and transmission. An algorithm that encodes the collected data with the lowest number of bits is a must in this case, as these nodes will have very limited storage capacity. Finally, the data will be stored in the volatile RAM in the device. 


\begin{tabular}{|c|}
\hline Storage Sub-Layer \\
\hline Encoding Sub-Layer \\
\hline Data Sampling and Quantizing Sub-Layer \\
\hline Environment Sensing Sub-Layer \\
\hline
\end{tabular}

Figure 5. Data acquisition layer

\subsection{Communication layer}

The communication layer will read the data stored in the RAM and re-code it suitable for transmission. Depending on the type available of the transmission technique, the data is to be coded that can withstand any adverse effects encountered during transmission. Mostly, wireless communication would be most feasible transmission technology used from distributed sensing nodes to central processing sites, the data will be traveling in very hostile environment with noise and interference from other sources. Also, the amount transmitted data must be as low as possible to reduce the transmission cost. Figure 6 shows the proposed architecture of the communication layer composed of error detection coding sub-layer, data compression sub-layer and transmission coding sub-layer.

\begin{tabular}{|c|c|}
\hline WiFi & 3G/4G \\
\hline Transmission Presentation Layer \\
\hline Transmission Coding Sub-Layer \\
\hline Data Compression Sub-Layer \\
\hline Error Detection Coding Sub-Layer \\
\hline
\end{tabular}

Figure 6. Transmission layer architecture

\subsection{Data analysis and knowledge generation layer}

The data analysis and knowledge generation layer will receive the data from different sensing nodes and first carry out an error detection and correction operation. Then the error corrected data will be combined and passed to the data analytics and knowledge layer that will create the usable information from the raw data received. Figure 7 shows the proposed data analysis and knowledge generation layer in detail in terms of its sub layers.

\begin{tabular}{|c|c|c|}
\hline \multicolumn{3}{|c|}{ Data Analytics \& Knowledge Generation Sub-Laye } \\
\hline \multicolumn{3}{|c|}{ Error Detection \& Correction Sub-Layer } \\
\hline \multicolumn{3}{|c|}{ Data Extraction Sub-Layer } \\
\hline \multicolumn{3}{|c|}{ Data Extraction and Presentation Sub-Layer } \\
\hline WiFi & $3 G / 4 G$ & Other \\
\hline
\end{tabular}

Figure 7. Data analysis and knowledge generation layer architecture

\subsection{Knowledge dissemination layer}

The knowledge dissemination layer is responsible for sending the knowledge generated to end users in the form of traffic advice. Also, the generated knowledge can be displayed on the web and LED based displays at prominent points within the city. Figure 8 shows the proposed knowledge dissemination layer in terms of its sub layers. 


\begin{tabular}{|c|c|c|}
\hline $\begin{array}{c}\text { SMS } \\
\text { Transmission }\end{array}$ & HTTP Protocol & $\begin{array}{c}\text { LED Display } \\
\text { Data Transmission }\end{array}$ \\
\hline SMS Generation & $\begin{array}{l}\text { HTML Code } \\
\text { Generation }\end{array}$ & $\begin{array}{l}\text { LED Display } \\
\text { Code Generation }\end{array}$ \\
\hline Data Coding 1 & Data Coding 2 & Data Coding 3 \\
\hline
\end{tabular}

Figure 8. Knowledge dissemination layer architecture

\subsection{Implementation of the integrated framework}

The implementation of the integrated framework needs to be separated between the central control and the environment sensing units. The data acquisition layer and the communication layer will be implemented within the sensing units while communication layer, data analysis \& knowledge generation layer and knowledge dissemination layer are to be implemented in the central control. Figure 9 shows the proposed implementation model of the proposed framework. The arrows within and outside the units show the data transfer paths within the units and between the environment sending unit and the central control.

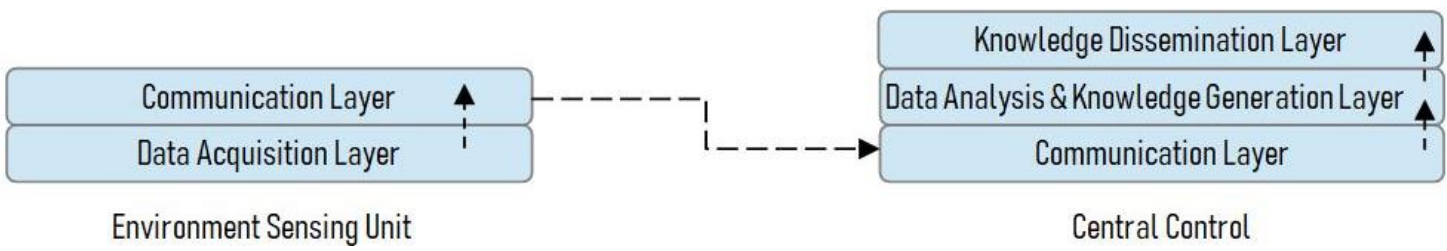

Figure 9. Implementation of the proposed model

The modular implementation and support for different communication technologies will enable the implementation of different sensing units with different technologies depending on the requirements. On the other hand, the data analysis and knowledge generation layer and the knowledge dissemination layer will be implemented in the central control. Data transmitted by the sensing units using different communication technologies can be received and combined by the central control for analysis and knowledge generation without any problem. Similar to the framework implemented in the sensing nodes, the central control also can take advantage of the future developments in the field. For example, when the new and advanced data analytics technologies become available, the entire system can be upgraded simply by replacing the functions within the data analytics and knowledge generation sub-layer without touching other parts of the system.

\section{DISCUSSION AND CONCLUSION}

This paper presented an integrated ICT model for environment aware traffic management with the main objective of reducing harmful pollution in critical regions. Traffic congestion is a major problem world over creating many harmful social, health and economic effects. Hence, different stakeholders including governments, and non-state sector actors focusing their efforts on managing traffic. Irrespective of the errors taken, the number of vehicles on the roads increases daily aggravating the already existing problems while creating new ones. The different parties in the community are affected differently by the traffic problem depending on various factors. This paper mainly concentrates on the negative health impacts on the marginal groups such as children, patients and older people and tries to reduce the pollution created by vehicles in areas where these groups tend to concentrate on especially near schools, hospitals and residential neighborhoods. This work takes advantage of new developments in the field of ICT including IoT, cloud computing, and advanced communication technologies. The proposed model can be effectively used as a reference framework for incorporating ICT into any traffic management implementation that involves pollution control as an integral component in it. The authors also propose that this framework can be further extended in the future incorporating other situations such disasters, accidents etc., to cover the entire spectrum of traffic management.

A framework for IoT-enabled environment aware traffic management (Mohamed Fazil Mohamed Firdhous) 


\section{ACKNOWLEDGEMENTS}

This research was supported by the Accelerating Higher Education Expansion and Development project of the Ministry of Higher Education of the Sri Lankan Government funded by the World Bank.

\section{REFERENCES}

[1] K. Farrell, "The Rapid Urban Growth Triad: A New Conceptual Framework for Examining the Urban Transition in Developing Countries," Sustainability, vol. 9, no. 8, pp. 1-19, 2017.

[2] M. Ali, et al., "The Dynamics of Traffic Congestion: A Specific Look into Malaysian Scenario and the Plausible Solutions to Eradicate it using Machine Learning," Indonesian Journal of Electrical Engineering and Computer Science (IJEECS), vol. 15, no. 2, pp. 1086-1094, 2019.

[3] A. Mohanty, et al., "Traffic Congestion Detection in a City Using Clustering Techniques in VANETs," Indonesian Journal of Electrical Engineering and Computer Science (IJEECS), vol. 13, no. 3, pp. 884-891, 2019.

[4] T. E. Parece, et al., "Assessing Urban Landscape Variables' Contributions to Microclimates," Advances in Meteorology, pp. 1-14, 2016.

[5] G. W. Leeson, "The Growth, Ageing and Urbanisation of Our World," Journal of Population Ageing, vol. 11, no. 2, pp. 107-115, 2018.

[6] J. R. Kenworthy, "Is Automobile Dependence in Emerging Cities an Irresistible Force? Perspectives from São Paulo, Taipei, Prague, Mumbai, Shanghai, Beijing, and Guangzhou," Sustainability, vol. 9, no. 11, pp. 1-30, 2017.

[7] W. M. H. W. Hussin, et al., "Review of Traffic Control Techniques for Emergency Vehicles," Indonesian Journal of Electrical Engineering and Computer Science (IJEECS), vol. 13, no. 3, pp. 1243-1251, 2019.

[8] V. Reddy, P. Gayathri, "Integration of Internet of Things with Wireless Sensor Network," International Journal of Electrical and Computer Engineering (IJECE), vol. 9, no. 1, pp. 439-444, 2019.

[9] M. Firdhous, et al., "Cloud Computing for Rural ICT Development: Opportunities and Challenges," 2013 International Conference on Computing, Electrical and Electronic Engineering (ICCEEE), Khartoum, Sudan, pp. 680-685, 2013.

[10] M. Firdhous, et al., "Honey Bee based Trust Management System for Cloud Computing," 3rd International Conference on Computing and Informatics (ICOCI 2011), Bandung, Indonesia, pp. 327-332, 2011.

[11] M. Firdhous, et al., "Fog Computing: Will it be the Future of Cloud Computing?" 3rd International Conference on Informatics \& Applications, Kuala Terengganu, Malaysia, pp. 8-15, 2014.

[12] R. Tiwari, "An Overview of Internet of Things (IoT): From Literature Survey to Application Implementation Perspective," International Research Journal of Engineering and Technology, vol. 4, no. 1, pp. 575-582, 2017.

[13] A. Al-Fuqaha, et al., "Internet of Things: A Survey on Enabling Technologies, Protocols and Applications," IEEE Communications Surveys \& Tutorials, vol. 17, no. 4, pp. 2347-2376, 2015.

[14] J. Gubbi, et al., "Internet of Things (IoT): A Vision, Architectural Elements, and Future Directions," Future Generation Computer Systems, vol. 29, no. 7, pp. 1645-1660, 2013.

[15] M. A. Razzaque, et al., "Middleware for Internet of Things: A Survey," IEEE Internet of Things Journal, vol. 3, no. 1, pp. 70-95, 2016.

[16] M. F. M. Firdhous, et al., "IoT Enabled Proactive Indoor Air Quality Monitoring System for Sustainable Health Management," 2nd International Conference on Computing and Communications Technologies, Chennai, India, pp. 216-221, 2017.

[17] S. M. Shirsath and N. B. Waghile, "IoT Based Smart Environmental Monitoring using Wireless Sensor Network," International Journal of Advanced Research in Electrical, Electronics and Instrumentation Engineering, vol. 7, no. 6, pp. 3023-3031, 2018

[18] R. Tanappagol and S. V. Kondikopp, "IoT Based Energy Efficient Environmental Monitoring Alerting and Controlling System," International Journal of Latest Technology in Engineering, Management \& Applied Science, vol. 6, no. 7, pp. 83-86, 2017.

[19] S. Shinde and V. N. Ghodke, "Design of WSN for Environmental Monitoring using IoT Application," International Journal of Innovative Research in Science, Engineering and Technology, vol. 5, no. 7, pp. 12963-12969, 2016.

[20] S. Zafar, et al., "An IoT Based Real-Time Environmental Monitoring System using Arduino and Cloud Service," Engineering, Technology \& Applied Science Research, vol. 8, no. 4, pp. 3238-3242, 2018.

[21] S. Kang, et al., "Laboratory Environment Monitoring: Implementation Experience and Field Study in a Tertiary General Hospital," Healthcare Informatics Research, vol. 24, no. 4, pp. 371-375, 2018.

[22] B. C. Kavitha, et al., "IoT Based Pollution Monitoring System using Raspberry-PI," International Journal of Pure and Applied Mathematics, vol. 118, no. 24, pp. 1-9, 2018.

[23] V. L. Paruchuri and P. Rajesh, "IoT for Monitoring Carbon Monoxide (CO) Emissions using Wireless Sensor Networks in Smart Cities," International Journal of Engineering \& Technology, vol. 7, no. 2.7, pp. 1045-1050, 2018.

[24] M. F. M. Firdhous and B. H. Sudantha, "\{Cloud, IoT $\}$-Powered Smart Weather Station for Microclimate Monitoring," Indonesian Journal of Electrical Engineering and Computer Science (IJEECS), vol. 17, no. 1, pp. 508-515, 2020.

[25] M. A. Suparman and S. L. Jong, "Automatic Smoke Detection System with Favoriot Platform using Internet of Things (IoT)," Indonesian Journal of Electrical Engineering and Computer Science (IJEECS), vol. 15, no. 2, pp. 1102-1108, 2019.

[26] H. F. Hawari, et al., "Development of Real Time Internet of Things (IoT) Based Air Quality Monitoring System," Indonesian Journal of Electrical Engineering and Computer Science (IJEECS), vol. 13, no. 3, pp. 1039-1047, 2019.

[27] Y. Huang, et al., "A Prototype IoT based Wireless Sensor Network for Traffic Information Monitoring," International Journal of Pavement Research and Technology, vol. 11, pp. 146-152, 2018.

Int J Elec \& Comp Eng, Vol. 11, No. 1, February 2021: 518 - 527 
[28] N. D. Matharia and S. P. Dave, "Smart Traffic Management System using IoT," International Journal of Computer Engineering and Applications, vol. XII, pp. 1-4, 2018.

[29] K. Bhagchandani and D. P. Augustine, "IoT based Heart Monitoring and Alerting System with Cloud Computing and Managing the Traffic for an Ambulance in India," International Journal of Electrical and Computer Engineering (IJECE), vol. 9, no. 6, pp. 5068-5074, 2019.

[30] N. Hashim, et al., "Automatic Traffic Light Controller for Emergency Vehicle using Peripheral Interface Controller," International Journal of Electrical and Computer Engineering (IJECE), vol. 9, no. 3, pp. 1788-1794, 2019.

[31] S. M. F. Malik, et al., "IoT Enabled Traffic Control Model using Raspberry PI," International Journal of Advanced Research in Computer Science, vol. 9, no. 3, pp. 157-160, 2018.

[32] Z. Dzulkurnain, et al., "Internet of Things (IoT) Based Traffic Management \& Routing Solution for Parking Space," Indonesian Journal of Electrical Engineering and Computer Science (IJEECS), vol. 15, no. 1, pp. 336-345, 2019.

[33] G. V. Vivekananda and P. C. Reddy, "Critical Analysis of Cross-layer Approach," International Conference on Green Computing and Internet of Things, Greater Noida, Delhi, India, pp. 12-16, 2015.

[34] J. Lopes, et al., "Traffic and Mobility Data Collection for Real-Time Applications," 13th International IEEE Conference on Intelligent Transportation Systems, Funchal, Portugal, pp. 216-223, 2010.

\section{BIOGRAPHIES OF AUTHORS}

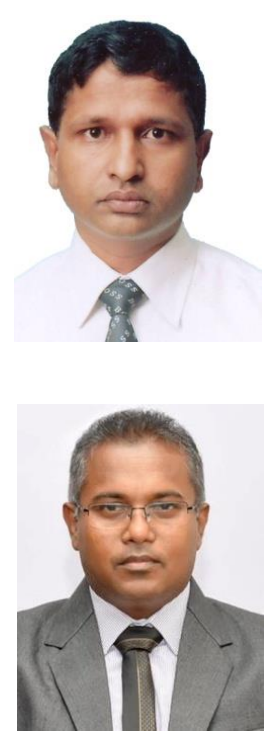

Dr. Mohamed Fazil Mohamed Firdhous is a Senior Lecturer attached to the Department of Information Technology of the Faculty of Information Technology, University of Moratwua, Sri Lanka. He is engaged in undergraduate and postgraduate teaching along with cutting edge research in the areas of trust and trust management in cloud computing, Internet of Things, mobile adhoc networks, vehicular networks, computer security, environment monitoring, intelligent transport systems and rural ICT development. He has teaching, research and industry experience in many countries including Sri Lanka, Singapore, United States of America and Malaysia. In addition to his teaching and research activities, he is a highly sought after ICT consultant to the government and private institutions in Sri Lanka.

Mr. B. H. Sudantha is the Dean of the Faculty of Information Technology and the Director, Information Technology Research Unit (ITRU) at the Faculty of Information Technology, University of Moratwua, Sri Lanka. Sudantha obtained his undergraduate and postgraduate degrees in Physics specializing in semiconductor devices at the University of Sri Jayewardenepura, Sri Lanka. He is currently engaged in undergraduate and postgraduate teaching in the broad areas of computer architecture with special emphasis on embedded processing. Sudantha's main research areas include environment monitoring, intelligent transport systems, mobile robotics and micro controller enabled device design.

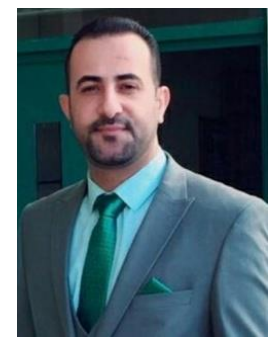

Dr. Naseer Ali Hussien is presently an Associate Professor and the Assistant Dean for Scientific Affairs of the Education College for Pure Sciences, Wasit University in Al Kut, Wasit, Iraq. He received his B.Sc. degree in Computer Science in 2000 from the Baghdad University in Baghdad, Iraq, his M.Sc. degree in Computer Science focusing on Computer Network and Communications from Hamdard University, Delhi, India in 2008 and the $\mathrm{PhD}$ in Information Technology from Universiti Utara Malaysia in 2013. His current research interests include network performance monitoring and analysis, wireless and mobile ad hoc networks, network protocol engineering, network simulation, network applications, smart cities, internet of things (IoT) and internet of vehicle (IoV). 